\title{
Analysis of broadband x-ray spectra of highly charged krypton from a microcalorimeter detector of an electron-beam ion trap
}

\author{
I. Kink, ${ }^{1}$ J. M. Laming, ${ }^{2}$ E. Takács,${ }^{3}$ J. V. Porto, ${ }^{1}$ J. D. Gillaspy, ${ }^{1}$ E. Silver, ${ }^{4}$ H. Schnopper, ${ }^{4}$ S. R. Bandler, ${ }^{4}$ M. Barbera, ${ }^{5}$ \\ N. Brickhouse, ${ }^{4}$ S. Murray ${ }^{4}$ N. Madden,${ }^{6}$ D. Landis,${ }^{6}$ J. Beeman, ${ }^{6}$ and E. E. Haller ${ }^{6}$ \\ ${ }^{1}$ National Institute of Standards and Technology, Gaithersburg, Maryland 20899 \\ ${ }^{2}$ Naval Research Laboratory, Code 7674L, Washington, DC 20375 \\ ${ }^{3}$ Massachusetts Institute of Technology, Cambridge, Massachusetts 02139 \\ ${ }^{4}$ Harvard-Smithsonian Center for Astrophysics, Cambridge, Massachusetts 02138 \\ ${ }^{5}$ Osservatorio Astronomico G. S. Vaiana, Palermo, Italy \\ ${ }^{6}$ Lawrence Berkeley National Laboratory, Berkeley, California 94720
}

(Received 13 June 2000; published 28 March 2001)

\begin{abstract}
Spectra of highly charged $\mathrm{Kr}$ ions, produced in an electron-beam ion trap (EBIT), have been recorded in a broad x-ray energy band $(0.3 \mathrm{keV}$ to $4 \mathrm{keV})$ with a microcalorimeter detector. Most of the spectral lines have been identified as transitions of B- to Al-like $\mathrm{Kr}$. The transition energies have been determined with $0.2 \%$ uncertainty. A semi-empirical EBIT plasma model has been created to calculate a synthetic spectrum of highly charged $\mathrm{Kr}$ and to determine a charge state distribution of $\mathrm{Kr}$ ions inside the EBIT.
\end{abstract}

DOI: 10.1103/PhysRevE.63.046409

PACS number(s): 52.70.- m, 32.30.Rj, 34.50.Fa, 34.80.Dp

\section{INTRODUCTION}

Laboratory plasmas produced from high $\mathrm{Z}$ gases have attracted interest in a number of applications. Laser-irradiated gaseous targets are routinely used for producing calibrated $\mathrm{x}$-ray sources. The gas target remains below the critical density of the laser, improving the efficiency with which laser light is absorbed, and converts much more of this energy to radiation, losing less to kinetic energy and conduction as in the cases with solid targets [1,2]. They also have advantages compared to solid targets in some potential technological applications (e.g., x-ray lithography [3]). High- $Z$ gases are also useful as "radiating divertors" in tokamaks. These create a buffer between the hot tokamak plasma and the tokamak chamber, thereby reducing particle fluxes and heat losses to the chamber walls (c.f. Ref. [4]). The investigation and application of plasma diagnostic potential of many of these applicable elements have largely been focused on $K$-shell emission (e.g., see Refs. [5,1]). However in principle the $L$ shells of these elements offer significant additional diagnostic potential [6]. In this paper we investigate line energies and relative intensities in ions of $\mathrm{Kr}$ between $\mathrm{B}$ - and Al-like.

Electron-beam ion traps (EBIT's) have proven to be versatile tools for investigating properties of very highly charged ions (see e.g., Ref. [7]). These properties are important for testing different theoretical atomic models as well as in a number of applications where high-temperature plasmas are involved. It is typical for these ions that a large fraction of the photon emission occurs in the $\mathrm{x}$-ray spectral region because of the fact that electron excitation energy increases with nuclear charge $Z$ [8]. Therefore spectroscopic investigations in the $\mathrm{x}$-ray region are important for determining the properties of highly charged ions. The majority of the experimental spectroscopic data in the x-ray region have so far been obtained with various types of crystal spectrometers. Although these instruments can provide the best resolving power necessary for precision studies, there are several limitations in their application for investigations of highly ionized plasmas. Their low transmission efficiency, which typically decreases with increasing spectral resolution, limits the resolving power typically to around thousands to tens of thousands. The narrow x-ray energy range that can be covered with one particular crystal sets limits to the studies where different parts of the spectra have to be compared, a situation that often occurs in the analysis of complex spectra of laboratory and astrophysical heavy ion plasmas, where lines from different charge states may overlap. Semiconductor detectors $[\mathrm{Si}(\mathrm{Li}), \mathrm{Ge}$, etc.] that can detect $\mathrm{x}$ rays in a broad energy band, on the other hand, have low-energy resolution (several hundred $\mathrm{eV}$ ), which is usually not sufficient for detailed spectroscopic investigations of highly charged ions.

An x-ray microcalorimeter $[9,10]$ is a recently developed type of $\mathrm{x}$-ray detector that has several unique properties that significantly improve spectroscopic measurements involving $\mathrm{x}$-ray photon detection. These instruments can cover a broad energy range ( 0.1 to $10 \mathrm{keV})$ with relatively high-energy resolution (about $6 \mathrm{eV}$ ) and close to $100 \%$ quantum efficiency. These properties make data acquisition more efficient and can provide information that is difficult or sometimes impossible to obtain by conventional methods. For example, since the spectra contain accurate line intensity information recorded over a broad spectral range, the comparison with a synthetic spectrum provided by plasma modeling calculations can yield information about various atomic and plasma parameters, e.g., excitation cross sections, electron and ion densities, etc. In this paper we demonstrate the microcalorimeter-EBIT combination in an investigation of highly ionized $\mathrm{Kr}$.

Until now, the experimental spectroscopic data in the x-ray region for highly charged $\mathrm{Kr}(q \geqslant 23+)$ ions have remained rather limited. In addition to pure atomic physics interest, these transitions have applications in radiative cooling experiments with tokamak plasmas by puffer gases 
$[11,12]$. The $\Delta n=0$ transitions within $n=2$ and $n=3$ configurations that occur in the extreme ultraviolet (EUV) regions have been studied by many groups with different techniques (e.g., Ref. [13-15]). However, the x-ray transitions from the $n=3$ levels to the ground state have been observed only by Burkhalter et al. [16] and Gordon et al. [17] who studied these transitions in the $\mathrm{F}-, \mathrm{Ne}-$ and $\mathrm{Na}$-like $\mathrm{Kr}$ ions produced in a $z$-pinch plasma. We determine line energies in the ions KrXXIV-XXXII, and identify some line ratios that have applications as electron density diagnostics for tokamak $\left(10^{12}-10^{14} \mathrm{~cm}^{-3}\right)$ or laser-plasma $\left(10^{19}-10^{21} \mathrm{~cm}^{-3}\right)$ sources.

In the following paragraphs details of the instrumentation, the plasma ionization balance modeling and spectral analysis will be summarized. The results of the line identifications of the observed $\mathrm{x}$-ray lines of $\mathrm{B}$ - to Al-like $\mathrm{Kr}$ as well as the charge state distribution of the krypton plasma will be presented, and finally some discussion of the electron-density sensitivity some of these lines are expected to exhibit is given.

\section{EXPERIMENTAL SETUP}

The experimental setup is thoroughly described in Refs. [18-20]. Here only a general outline is given. The $\mathrm{Kr}$ ions are radially confined inside the EBIT trap region by the combination of an electric field due to an intense beam of electrons and a homogeneous strong magnetic field parallel to the electron beam. Axially the ions are trapped by an electric field created by a set of cylindrical drift tube electrodes. The electron beam is compressed by a $3 \mathrm{~T}$ magnetic field that is generated with a superconducting coil to achieve maximum current density. In our measurements the beam current was typically $115 \mathrm{~mA}$ with an estimated radius of $35 \mu \mathrm{m}$ (current density $\approx 3 \mathrm{kA} / \mathrm{cm}^{2}$ ). The beam energy (typically in the few $\mathrm{keV}$ range) was one of the adjustable experimental parameters. The krypton gas was let into the trap using a gas injection system attached to the EBIT [21]. By varying the neutral gas pressure inside the EBIT the charge state distribution and dynamics can be manipulated [22] due to shifts in the balance between ionization and recombination processes.

The trap was dumped every $1.35 \mathrm{~s}$ to prevent a slow buildup of contaminating heavy ions, small amounts of which are present inside the EBIT despite the ultrahigh vacuum conditions (typically $\approx 10^{-7} \mathrm{~Pa}$ ).

Collisions of $\mathrm{Kr}$ atoms and ions with beam electrons lead to gradual ionization and excitation of the $\mathrm{Kr}$ ions. The $\mathrm{x}$ rays emitted in the region of $0.3 \mathrm{keV}$ to $10 \mathrm{keV}$ were collected with a focusing x-ray optic $[23,24]$ and detected by a neutron transmutation-doped germanium-based microcalorimeter $[10,20]$, where the increase of the temperature of the detector, that is proportional to the energy of the absorbed photon, is recorded. The x-ray optic and detector were separated from the EBIT with a thin $(20 \mathrm{~nm})$ mylar window to prevent contamination of the vacuum inside the EBIT. The transmission characteristics of the x-ray optic and a description of the detector can be found in Ref. [20]. Over the spectral region where the analyzed Kr lines appear, the combination of the total transmission of the optical components and
TABLE I. Experimental conditions for $\mathrm{Kr}$ spectra.

\begin{tabular}{lcc}
\hline \hline Set & $\begin{array}{c}\text { Beam energy } \\
(\mathrm{keV})\end{array}$ & $\begin{array}{c}\text { Gas injection pressure } \\
\left(\times 10^{-4} \mathrm{~Pa}\right)\end{array}$ \\
\hline I & 5.77 & 1.33 \\
II & 12.44 & 1.33 \\
III & 6.06 & 11.7 \\
IV & 6.06 & 0.5 \\
\hline
\end{tabular}

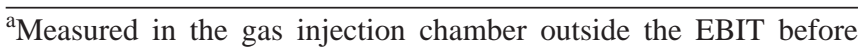
expansion into the low-pressure region at the trap center [21].

the efficiency of the microcalorimeter is a smooth and flat function of the photon energy, and therefore no corrections to the line intensities have been made. Four recordings with $\mathrm{Kr}$ gas were taken (Table I), varying the gas pressure and electron-beam energy. The data acquisition time for each spectrum was about $20 \mathrm{~min}$. Spectra of highly charged N, Ar, and $\mathrm{O}$ were also recorded under identical experimental conditions for detector energy calibration purposes.

\section{DATA ANALYSIS}

Experimental spectra were calibrated using He-like $\mathrm{N}$ [25,26] and $\mathrm{Ar}$ [27], H-like $\mathrm{N}$ and $\mathrm{O}$ [28], and Ne- and F-like $\mathrm{Kr}$ [16] lines. Altogether, 15 reference lines were used that spread over the large energy interval of $300 \mathrm{eV}$ to $4000 \mathrm{eV}$. Three groups of lines are well distinguished in the spectra: $\mathrm{N}$ and $\mathrm{O}$ lines at the low-energy side, $\mathrm{Kr}$ lines in the center, and Ar lines at the high-energy side. Polynomial functions were used for energy calibration and the best fit was achieved with a third degree polynomial. The weighted average scattering of reference lines was $1.4 \mathrm{eV}$, where inverse squares of the uncertainties of the reference lines (mainly due to statistical uncertainty in the line positions) were used as the weights.

The spectral lines were first fitted with a sum of Gaussian profiles to get accurate line positions using the computer code GFIT [29]. The typical full width at half maximum was 2.5 data channels, corresponding to approximately $6 \mathrm{eV}$. The statistical uncertainty (one standard deviation) of the line position was typically $0.5 \mathrm{eV}$ to $1.0 \mathrm{eV}$ which, taking into account the accuracy of the calibration, gives an uncertainty of $1.7 \mathrm{eV}$ for identified single lines. Since most of the lines are blended with close-lying lines we quote $3.5 \mathrm{eV}$ as an estimate of the combined standard uncertainty of transition energies. This value includes the statistical uncertainty (uniform distribution) in the line position of $3.2 \mathrm{eV}$ and the calibration uncertainty of $1.4 \mathrm{eV}$. The final energies that are presented in Table II are the averages from the four different measurements. The quoted uncertainty agrees well with the statistical deviation of transition energies from four different measurements (average $\approx 2.5 \mathrm{eV}$ ) indicating that there are no significant systematic deviations between different measurements. In Fig. 1, the part of the spectrum where the Kr lines appear is shown. This spectral structure contains lines of Alto Be-like $\mathrm{Kr}$ ions. Most of the strong lines come from the $\Delta n=1$ transitions between $n=2$ and $n=3$ configurations ( $L$ transitions). The electronic structure of ions with many elec- 
TABLE II. Measured and calculated energies of $E 1, E 2$, and $M 1$ transitions in B-like to Al-like $\mathrm{Kr}$ Ions.

\begin{tabular}{|c|c|c|c|c|c|c|}
\hline$I_{\text {exp }}^{\mathrm{a}}$ & $E_{\text {exp }}(\mathrm{eV})^{\mathrm{b}}$ & $I_{t h}{ }^{\mathrm{c}}$ & $E_{t h}(\mathrm{eV})^{\mathrm{d}}$ & Lower level & Upper level & $\begin{array}{l}\text { Leading } \\
\text { eigenfunction } \\
\text { contribution }\end{array}$ \\
\hline
\end{tabular}

B-like $\left(2 s^{2} 2 p^{2} \mathrm{P}^{\circ}{ }_{1 / 2}\right)$

\begin{tabular}{|c|c|c|c|c|c|c|c|c|}
\hline \multirow[t]{2}{*}{7} & \multirow[t]{2}{*}{2230.3} & 1.9 & 2231.9 & \multirow[t]{2}{*}{$2 p$} & \multirow[t]{2}{*}{${ }^{2} \mathrm{P}^{\circ}{ }_{1 / 2}$} & \multirow[t]{2}{*}{$2 s 2 p 3 p$} & \multirow{2}{*}{$\begin{array}{l}(1,3 / 2)_{3 / 2} \\
(1,3 / 2)_{1 / 2}\end{array}$} & \multirow{2}{*}{$\begin{array}{l}89 \% \\
90 \%\end{array}$} \\
\hline & & 1.1 & 2229.5 & & & & & \\
\hline 1 & 2223.9 & 0.7 & 2217.9 & & & & $(0,3 / 2)_{3 / 2}$ & $93 \%$ \\
\hline 22 & 2183.7 & 7.1 & 2187.8 & & & $3 d$ & ${ }^{2} \mathrm{D}_{3 / 2}$ & $100 \%$ \\
\hline \multirow[t]{2}{*}{1} & 2126.1 & 0.5 & 2127.7 & $2 s 2 p^{2}$ & ${ }^{4} \mathrm{P}_{3 / 2}$ & $2 s 2 p 3 d$ & $(0,5 / 2)^{\circ}{ }_{5 / 2}$ & $45 \%$ \\
\hline & & 1.1 & 2126.6 & $2 p$ & ${ }^{2} \mathrm{P}^{\circ}{ }_{3 / 2}$ & $3 d$ & ${ }^{2} \mathrm{D}_{3 / 2}$ & $100 \%$ \\
\hline \multirow[t]{2}{*}{30} & 2089.1 & 2.4 & 2090.1 & $2 s 2 p^{2}$ & ${ }^{2} \mathrm{D}_{3 / 2}$ & $2 s 2 p 3 d$ & $(1,5 / 2)^{\circ}{ }_{5 / 2}$ & $72 \%$ \\
\hline & & 1.4 & 2079.2 & & ${ }^{2} \mathrm{P}_{1 / 2}$ & $2 s 2 p 3 d$ & $(1,5 / 2)^{\circ}{ }_{3 / 2}$ & $49 \%$ \\
\hline 18 & 2010.7 & 1.5 & 2010.1 & & ${ }^{2} \mathrm{D}_{3 / 2}$ & $2 s 2 p 3 s$ & $(1,1 / 2)^{\circ}{ }_{1 / 2}$ & $81 \%$ \\
\hline 31 & 1995.7 & 0.8 & 2001.0 & & ${ }^{2} \mathrm{P}_{1 / 2}$ & & & \\
\hline
\end{tabular}

C-like $\left(2 s^{2} 2 p^{2}{ }^{3} \mathrm{P}_{0}\right)$

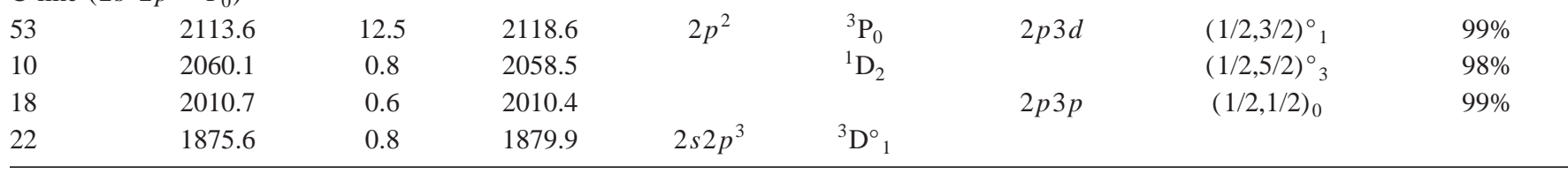

\begin{tabular}{|c|c|c|c|c|c|c|c|c|}
\hline \multicolumn{9}{|c|}{ N-like $\left(2 s^{2} 2 p^{3}{ }^{4} \mathrm{~S}_{3 / 2}^{\circ}\right)$} \\
\hline 10 & 2060.1 & 2.7 & 2055.1 & $2 p^{3}$ & ${ }^{4} \mathrm{~S}_{3 / 2}^{\circ}$ & $2 p^{2}\left({ }^{3} \mathrm{P}\right) 3 d$ & $(2,3 / 2)_{1 / 2}$ & $48 \%$ \\
\hline \multirow[t]{2}{*}{44} & 2047.8 & 4.2 & 2053.0 & & & $2 p^{2}\left({ }^{1} \mathrm{D}\right) 3 d$ & $(2,3 / 2)_{3 / 2}$ & $43 \%$ \\
\hline & & 2.9 & 2051.3 & & & $2 p^{2}\left({ }^{3} \mathrm{P}\right) 3 d$ & $(2,3 / 2)_{5 / 2}$ & $26 \%$ \\
\hline \multirow[t]{2}{*}{1} & 2035.8 & 1.1 & 2033.8 & & & & $(1,3 / 2)_{5 / 2}$ & $66 \%$ \\
\hline & & 0.7 & 2031.8 & & & & $(1,3 / 2)_{3 / 2}$ & $94 \%$ \\
\hline 18 & 2010.7 & 1.3 & 2012.0 & & ${ }^{2} \mathrm{D}_{3 / 2}^{\circ}$ & $2 p^{2}\left({ }^{1} \mathrm{D}\right) 3 d$ & $(2,5 / 2)_{5 / 2}$ & $34 \%$ \\
\hline \multirow[t]{3}{*}{31} & 1995.7 & 1.3 & 1995.2 & & & $2 p^{2}\left({ }^{3} \mathrm{P}\right) 3 d$ & $(1,5 / 2)_{5 / 2}$ & $81 \%$ \\
\hline & & 4.6 & 1994.9 & & ${ }^{4} \mathrm{~S}_{3 / 2}^{\circ}$ & & $(0,5 / 2)_{5 / 2}$ & $75 \%$ \\
\hline & & 0.6 & 1992.0 & & ${ }^{2} \mathrm{D}^{\circ}{ }_{5 / 2}$ & $2 p^{2}\left({ }^{1} \mathrm{D}\right) 3 d$ & $(2,3 / 2)_{3 / 2}$ & $43 \%$ \\
\hline \multirow[t]{2}{*}{1} & 1986.4 & 1.6 & 1990.1 & & & $2 p^{2}\left({ }^{3} \mathrm{P}\right) 3 d$ & $(2,3 / 2)_{5 / 2}$ & $26 \%$ \\
\hline & & 0.8 & 1985.6 & & ${ }^{4} \mathrm{~S}^{\circ}{ }_{3 / 2}$ & & $(0,3 / 2)_{3 / 2}$ & $79 \%$ \\
\hline 1 & 1941.1 & 0.5 & 1942.7 & & & $2 p^{2}\left({ }^{1} \mathrm{D}\right) 3 s$ & $(2,1 / 2)_{5 / 2}$ & $51 \%$ \\
\hline 17 & 1788.2 & 0.8 & 1790.3 & $2 s 2 p^{4}$ & ${ }^{4} \mathrm{P}_{3 / 2}$ & $2 p^{2}\left({ }^{3} \mathrm{P}\right) 3 p$ & $(2,1 / 2)^{\circ}{ }_{3 / 2}$ & $30 \%$ \\
\hline \multicolumn{9}{|c|}{ O-like $\left(2 s^{2} 2 p^{4}{ }^{3} \mathrm{P}_{2}\right)$} \\
\hline \multirow[t]{2}{*}{31} & 1995.7 & 0.7 & 1992.0 & $2 p^{4}$ & ${ }^{3} \mathrm{P}_{2}$ & $2 p^{3}\left({ }^{2} \mathrm{D}^{\circ}\right) 3 d$ & ${ }^{3} \mathrm{~S}_{1}^{\circ}$ & $63 \%$ \\
\hline & & 2.8 & 1991.0 & & ${ }^{3} \mathrm{P}_{0}$ & $2 p^{3}\left({ }^{2} \mathrm{P}^{\circ}\right) 3 d$ & ${ }^{3} \mathrm{D}^{\circ}{ }_{1}$ & $47 \%$ \\
\hline \multirow[t]{4}{*}{1} & 1986.4 & 0.9 & 1987.2 & & ${ }^{3} \mathrm{P}_{2}$ & $2 p^{3}\left({ }^{2} \mathrm{D}^{\circ}\right) 3 d$ & ${ }^{3} \mathrm{P}^{\circ}{ }_{1}$ & $50 \%$ \\
\hline & & 2.9 & 1987.2 & & & & ${ }^{3} \mathrm{D}_{2}^{\circ}$ & $59 \%$ \\
\hline & & 0.7 & 1986.6 & & ${ }^{1} \mathrm{D}_{2}$ & $2 p^{3}\left({ }^{2} \mathrm{P}^{\circ}\right) 3 d$ & ${ }^{3} \mathrm{D}^{\circ}{ }_{3}$ & $44 \%$ \\
\hline & & 0.7 & 1985.6 & & & & ${ }^{1} \mathrm{~F}_{3}^{\circ}$ & $49 \%$ \\
\hline \multirow[t]{2}{*}{46} & 1981.5 & 7.5 & 1984.0 & & ${ }^{3} \mathrm{P}_{2}$ & $2 p^{3}\left({ }^{2} \mathrm{D}^{\circ}\right) 3 d$ & ${ }^{3} \mathrm{D}_{3}^{\circ}$ & $50 \%$ \\
\hline & & 0.6 & 1979.3 & & & & ${ }^{3} \mathrm{P}_{2}^{\circ}$ & $30 \%$ \\
\hline 1 & 1973.5 & 1.4 & 1968.6 & & & & ${ }^{3} \mathrm{G}^{\circ}{ }_{3}$ & $42 \%$ \\
\hline \multirow[t]{2}{*}{12} & 1961.9 & 1.0 & 1966.1 & & & & ${ }^{3} \mathrm{~F}_{3}^{\circ}$ & $44 \%$ \\
\hline & & 1.1 & 1964.5 & & & & ${ }^{3} \mathrm{~F}_{2}^{\circ}$ & $41 \%$ \\
\hline 1 & 1941.1 & 0.7 & 1939.9 & & ${ }^{1} \mathrm{D}_{2}$ & $2 p^{3}\left({ }^{2} \mathrm{P}^{\circ}\right) 3 d$ & ${ }^{3} \mathrm{~F}_{3}^{\circ}$ & $51 \%$ \\
\hline \multirow[t]{3}{*}{32} & 1932.4 & 0.7 & 1935.4 & & ${ }^{3} \mathrm{P}_{1}$ & $2 p^{3}\left({ }^{2} \mathrm{D}^{\circ}\right) 3 d$ & ${ }^{3} \mathrm{P}_{1}^{\circ}$ & $50 \%$ \\
\hline & & 0.9 & 1935.4 & & & & ${ }^{3} \mathrm{D}^{\circ}{ }_{2}$ & $59 \%$ \\
\hline & & 6.7 & 1934.2 & & ${ }^{3} \mathrm{P}_{2}$ & $2 p^{3}\left({ }^{4} S^{\circ}\right) 3 d$ & ${ }^{3} \mathrm{D}^{\circ}{ }_{3}$ & $26 \%$ \\
\hline
\end{tabular}


TABLE II. (Continued).

\begin{tabular}{|c|c|c|c|c|c|c|c|c|}
\hline \multirow[t]{2}{*}{$I_{\text {exp }}{ }^{\mathrm{a}}$} & \multirow[t]{2}{*}{$E_{\text {exp }}(\mathrm{eV})^{\mathrm{b}}$} & \multirow{2}{*}{$\begin{array}{r}I_{t h}{ }^{\mathrm{c}} \\
1.2\end{array}$} & \multirow{2}{*}{$\begin{array}{c}E_{t h}(\mathrm{eV})^{\mathrm{d}} \\
1930.6\end{array}$} & \multicolumn{2}{|c|}{ Lower level } & \multicolumn{2}{|c|}{ Upper level } & \multirow{2}{*}{$\begin{array}{c}\begin{array}{c}\text { Leading } \\
\text { eigenfunction } \\
\text { contribution }\end{array} \\
63 \%\end{array}$} \\
\hline & & & & & ${ }^{1} \mathrm{D}_{2}$ & $2 p^{3}\left({ }^{2} \mathrm{D}^{\circ}\right) 3 d$ & ${ }^{1} \mathrm{~F}_{3}^{\circ}$ & \\
\hline \multirow[t]{4}{*}{38} & 1925.2 & 1.1 & 1927.3 & & ${ }^{3} \mathrm{P}_{1}$ & & ${ }^{3} \mathrm{P}^{\circ}{ }_{2}$ & $30 \%$ \\
\hline & & 0.8 & 1926.7 & & ${ }^{1} \mathrm{D}_{2}$ & & ${ }^{3} \mathrm{~S}^{\circ}{ }_{1}$ & $63 \%$ \\
\hline & & 2.2 & 1924.9 & & ${ }^{3} \mathrm{P}_{2}$ & $2 p^{3}\left({ }^{4} \mathrm{~S}^{\circ}\right) 3 d$ & ${ }^{5} \mathrm{D}^{\circ}{ }_{2}$ & $24 \%$ \\
\hline & & 0.9 & 1924.3 & & & $2 p^{3}\left({ }^{2} \mathrm{D}^{\circ}\right) 3 p$ & ${ }^{3} \mathrm{~F}_{4}$ & $100 \%$ \\
\hline \multirow[t]{3}{*}{42} & 1917.5 & 0.5 & 1918.3 & & & $2 p^{3}\left({ }^{4} \mathrm{~S}^{\circ}\right) 3 d$ & ${ }^{5} \mathrm{D}^{\circ}{ }_{1}$ & $51 \%$ \\
\hline & & 0.8 & 1917.1 & & & & ${ }^{5} \mathrm{D}^{\circ}{ }_{3}$ & $39 \%$ \\
\hline & & 2.2 & 1917.1 & & ${ }^{3} \mathrm{P}_{0}$ & & ${ }^{3} \mathrm{D}^{\circ}{ }_{1}$ & $45 \%$ \\
\hline 22 & 1875.6 & 0.6 & 1877.9 & & ${ }^{3} \mathrm{P}_{1}$ & $2 p^{3}\left({ }^{2} \mathrm{P}^{\circ}\right) 3 s$ & ${ }^{3} \mathrm{P}_{2}^{\circ}$ & $59 \%$ \\
\hline \multirow[t]{2}{*}{24} & 1861.5 & 2.7 & 1864.4 & & ${ }^{3} \mathrm{P}_{2}$ & $2 p^{3}\left({ }^{2} \mathrm{D}^{\circ}\right) 3 s$ & ${ }^{3} \mathrm{D}^{\circ}{ }_{3}$ & $100 \%$ \\
\hline & & 0.6 & 1864.4 & & ${ }^{1} \mathrm{D}_{2}$ & $2 p^{3}\left({ }^{2} \mathrm{P}^{\circ}\right) 3 s$ & ${ }^{3} \mathrm{P}^{\circ}{ }_{2}$ & $59 \%$ \\
\hline 44 & 1854.1 & 1.3 & 1851.0 & & ${ }^{3} \mathrm{P}_{2}$ & $2 p^{3}\left({ }^{4} \mathrm{~S}^{\circ}\right) 3 s$ & ${ }^{5} \mathrm{~S}_{2}^{\circ}$ & $40 \%$ \\
\hline \multirow[t]{4}{*}{50} & 1803.9 & 1.3 & 1809.7 & & & & ${ }^{3} \mathrm{~S}^{\circ}{ }_{1}$ & $40 \%$ \\
\hline & & 2.1 & 1803.9 & & & & ${ }^{5} \mathrm{~S}_{2}^{\circ}$ & $48 \%$ \\
\hline & & 0.6 & 1802.8 & & ${ }^{3} \mathrm{P}_{1}$ & $2 p^{3}\left({ }^{2} \mathrm{D}^{\circ}\right) 3 s$ & ${ }^{3} \mathrm{D}^{\circ}{ }_{1}$ & $51 \%$ \\
\hline & & 1.1 & 1802.6 & & ${ }^{1} \mathrm{D}_{2}$ & & ${ }^{1} \mathrm{D}_{2}^{\circ}$ & $66 \%$ \\
\hline 36 & 1797.0 & 0.6 & 1799.2 & & & & ${ }^{3} \mathrm{D}_{3}^{\circ}$ & $100 \%$ \\
\hline \multicolumn{9}{|c|}{ F-like $\left(2 s^{2} 2 p^{5}{ }^{2} \mathrm{P}_{3 / 2}^{\circ}\right)$} \\
\hline 20 & 2025.3 & 0.7 & 2020.2 & $2 s^{2} 2 p^{5}$ & ${ }^{2} \mathrm{P}_{3 / 2}^{\circ}$ & $2 s 2 p^{5} 3 p$ & $(1,3 / 2)_{5 / 2}$ & $82 \%$ \\
\hline \multirow[t]{2}{*}{$18^{\mathrm{f}}$} & 2010.7 & 0.6 & 2009.8 & & & & $(2,3 / 2)_{1 / 2}$ & $50 \%$ \\
\hline & & 0.6 & 2007.5 & & & & $(1,1 / 2)_{3 / 2}$ & $74 \%$ \\
\hline \multirow[t]{2}{*}{31} & 1995.7 & 0.8 & 1999.7 & & & & $(2,3 / 2)_{5 / 2}$ & $99 \%$ \\
\hline & & 0.6 & 1996.8 & & & & $(2,3 / 2)_{3 / 2}$ & $82 \%$ \\
\hline $46^{\mathrm{f}}$ & 1981.5 & 0.6 & 1983.4 & & & & $(2,1 / 2)_{5 / 2}$ & $99 \%$ \\
\hline \multirow[t]{4}{*}{$42^{f}$} & 1917.5 & 10.0 & 1919.5 & & & $2 s^{2} 2 p^{4}\left({ }^{1} \mathrm{D}\right) 3 d$ & $(2,3 / 2)_{5 / 2}$ & $64 \%$ \\
\hline & & 5.6 & 1918.0 & & & & $(2,3 / 2)_{3 / 2}$ & $36 \%$ \\
\hline & & 0.6 & 1914.8 & & & & $(2,5 / 2)_{5 / 2}$ & $80 \%$ \\
\hline & & 2.8 & 1914.8 & & & & $(2,3 / 2)_{1 / 2}$ & $60 \%$ \\
\hline \multirow[t]{2}{*}{$1^{\mathrm{f}}$} & 1900.7 & 2.1 & 1903.6 & & & $2 s^{2} 2 p^{4}\left({ }^{3} \mathrm{P}\right) 3 d$ & $(1,5 / 2)_{5 / 2}$ & $77 \%$ \\
\hline & & 2.3 & 1901.9 & & & & $(1,3 / 2)_{5 / 2}$ & $63 \%$ \\
\hline \multirow[t]{3}{*}{$22^{f}$} & 1875.6 & 0.6 & 1876.2 & & ${ }^{2} \mathrm{P}_{1 / 2}^{\circ}$ & $2 s^{2} 2 p^{4}\left({ }^{1} \mathrm{D}\right) 3 d$ & $(2,5 / 2)_{1 / 2}$ & $51 \%$ \\
\hline & & 1.4 & 1873.4 & & & & $(2,3 / 2)_{3 / 2}$ & $30 \%$ \\
\hline & & 4.2 & 1873.1 & & ${ }^{2} \mathrm{P}_{3 / 2}^{\circ}$ & $2 s^{2} 2 p^{4}\left({ }^{3} \mathrm{P}\right) 3 d$ & $(0,5 / 2)_{5 / 2}$ & $47 \%$ \\
\hline $33^{f}$ & 1869.7 & 2.1 & 1869.4 & & & & $(0,3 / 2)_{3 / 2}$ & $41 \%$ \\
\hline \multirow[t]{3}{*}{$24^{\mathrm{f}}$} & 1861.5 & 0.9 & 1863.0 & & ${ }^{2} \mathrm{P}_{1 / 2}^{\circ}$ & $2 s^{2} 2 p^{4}\left({ }^{1} \mathrm{D}\right) 3 d$ & $(2,3 / 2)_{3 / 2}$ & $36 \%$ \\
\hline & & 6.8 & 1861.9 & & ${ }^{2} \mathrm{P}^{\circ}{ }_{3 / 2}$ & $2 s^{2} 2 p^{4}\left({ }^{3} \mathrm{P}\right) 3 d$ & $(2,5 / 2)_{5 / 2}$ & $68 \%$ \\
\hline & & 3.4 & 1858.2 & & & & $(2,5 / 2)_{3 / 2}$ & $57 \%$ \\
\hline $44^{\mathrm{f}}$ & 1854.1 & 1.4 & 1853.8 & & & & $(2,5 / 2)_{1 / 2}$ & $56 \%$ \\
\hline $56^{\mathrm{f}}$ & 1847.0 & 0.7 & 1842.0 & $2 s 2 p^{6}$ & ${ }^{2} S_{1 / 2}$ & $2 s 2 p^{5} 3 d$ & $(1,3 / 2)^{\circ}{ }_{3 / 2}$ & $51 \%$ \\
\hline $16^{\mathrm{f}}$ & 1783.1 & 1.7 & 1785.5 & $2 s^{2} 2 p^{5}$ & ${ }^{2} \mathrm{P}_{3 / 2}^{\circ}$ & $2 s^{2} 2 p^{4}\left({ }^{1} \mathrm{D}\right) 3 s$ & $(2,1 / 2)_{5 / 2}$ & $78 \%$ \\
\hline \multirow[t]{2}{*}{$12^{f}$} & 1771.5 & 1.0 & 1771.4 & & & $2 s^{2} 2 p^{4}\left({ }^{3} \mathrm{P}\right) 3 s$ & $(1,1 / 2)_{3 / 2}$ & $99 \%$ \\
\hline & & 0.9 & 1769.9 & & & $2 s^{2} 2 p^{4}\left({ }^{3} \mathrm{P}\right) 3 p$ & $(2,1 / 2)^{\circ}{ }_{5 / 2}$ & $76 \%$ \\
\hline \multirow[t]{2}{*}{$1^{\mathrm{f}}$} & 1735.6 & 0.6 & 1742.3 & & & $2 s^{2} 2 p^{4}\left({ }^{3} \mathrm{P}\right) 3 s$ & $(0,1 / 2)_{1 / 2}$ & $51 \%$ \\
\hline & & 0.5 & 1732.1 & & ${ }^{2} \mathrm{P}^{\circ}{ }_{1 / 2}$ & $2 s^{2} 2 p^{4}\left({ }^{1} \mathrm{D}\right) 3 s$ & $(2,1 / 2)_{3 / 2}$ & $74 \%$ \\
\hline $21^{\mathrm{f}}$ & 1722.6 & 2.1 & 1724.1 & & ${ }^{2} \mathrm{P}_{3 / 2}^{\circ}$ & $2 s^{2} 2 p^{4}\left({ }^{3} \mathrm{P}\right) 3 s$ & $(2,1 / 2)_{3 / 2}$ & $75 \%$ \\
\hline $17^{\mathrm{f}}$ & 1716.1 & 3.0 & 1719.1 & & & & $(2,1 / 2)_{5 / 2}$ & $79 \%$ \\
\hline & & 1.3 & 1718.9 & $2 s 2 p^{6}$ & ${ }^{2} \mathrm{~S}_{1 / 2}$ & $2 s 2 p^{5} 3 s$ & $(1,1 / 2)^{\circ}{ }_{3 / 2}$ & $75 \%$ \\
\hline 1 & 1697.2 & 1.2 & 1699.3 & & & & $(2,1 / 2)^{\circ}{ }_{3 / 2}$ & $93 \%$ \\
\hline 7 & 1615.8 & 2.7 & 1611.2 & & & $2 s^{2} 2 p^{4}\left({ }^{1} \mathrm{D}\right) 3 p$ & $(2,1 / 2)^{\circ} 3 / 2$ & $32 \%$ \\
\hline
\end{tabular}


TABLE II. (Continued).

\begin{tabular}{|c|c|c|c|c|c|c|}
\hline$I_{\text {exp }}{ }^{\mathrm{a}}$ & $E_{\text {exp }}(\mathrm{eV})^{\mathrm{b}}$ & $I_{t h}{ }^{\mathrm{c}}$ & $E_{t h}(\mathrm{eV})^{\mathrm{d}}$ & Lower level & Upper level & $\begin{array}{c}\text { Leading } \\
\text { eigenfunction } \\
\text { contribution }\end{array}$ \\
\hline
\end{tabular}

Ne-like $\left(2 s^{2} 2 p^{6}{ }^{1} \mathrm{~S}_{0}\right)$

12035.8

$12^{\mathrm{f}} \quad 1961.9$

$\begin{array}{ll}12^{\mathrm{f}} & 1961.9 \\ 1^{\mathrm{f}} & 1941.1\end{array}$

$1.4 \quad 2034.8$

$2 p^{6} \quad{ }^{1} \mathrm{~S}_{0}$

$2 s 2 p^{6} 3 d$
$2 s 2 p^{6} 3 p$

${ }^{1} \mathrm{D}_{2} \quad 97 \%$

$44^{\mathrm{f}} \quad 1854.1$

$3.1 \quad 1960.5$

1945.4

1.1

$50^{\mathrm{f}} \quad 1803.9$

$28.0 \quad 1852.7$

$12 \quad 1771.5$

23.0

1803.4

1722.6

0.7

1772.7

21

0.7

1720.1

$31^{\mathrm{f}} \quad 1705.5$

$\begin{array}{ll}6.7 & 1705.9\end{array}$

$44^{\mathrm{f}}$

1651.5

1703.7

1652.4

$\begin{array}{cc}11.8 & 1652.4 \\ 5.9 & 1649.1\end{array}$

$2 p^{5} 3 d$
$2 p^{5} 3 p$
$2 p^{5} 3 s$
$2 p^{5} 3 p$
$2 p^{5} 3 s$

${ }^{1} \mathrm{P}^{\circ}{ }_{1}$

$76 \%$

${ }^{3} \mathrm{P}^{\circ}{ }_{1} \quad 76 \%$

${ }^{1} \mathrm{P}^{\circ}{ }_{1} \quad 53 \%$

${ }^{3} \mathrm{D}^{\circ}{ }_{1} \quad 46 \%$

${ }^{3} \mathrm{D}_{2} \quad 47 \%$

$\begin{array}{ll}{ }^{3} \mathrm{D}_{2} & 67 \%\end{array}$

${ }^{3} \mathrm{P}^{\circ}{ }_{1} \quad 63 \%$

${ }^{3} \mathrm{D}_{2} \quad 53 \%$

${ }^{1} \mathrm{P}^{\circ}{ }_{1} \quad 63 \%$

${ }^{3} \mathrm{P}^{\circ}{ }_{2} \quad 100 \%$

\begin{tabular}{|c|c|c|c|c|c|c|c|c|}
\hline \multicolumn{9}{|c|}{ Na-like $\left(2 s^{2} 2 p^{6} 3 s^{2} \mathrm{~S}_{1 / 2}\right)$} \\
\hline 56 & 1847.0 & 14.4 & 1841.1 & \multirow[t]{4}{*}{$2 p^{6} 3 s$} & \multirow[t]{4}{*}{${ }^{2} S_{1 / 2}$} & \multirow[t]{4}{*}{$2 p^{5} 3 s 3 d$} & $(1,3 / 2)^{\circ}{ }_{3 / 2}$ & $43 \%$ \\
\hline $13^{\mathrm{f}}$ & 1834.0 & 9.6 & 1836.8 & & & & $(1,3 / 2)^{\circ}{ }_{1 / 2}$ & $95 \%$ \\
\hline \multirow[t]{2}{*}{17} & 1788.2 & 15.0 & 1790.3 & & & & $(2,5 / 2)^{\circ} 3 / 2$ & $47 \%$ \\
\hline & & 7.3 & 1789.1 & & & & $(2,5 / 2)^{\circ}{ }_{1 / 2}$ & $88 \%$ \\
\hline
\end{tabular}

Mg-like $\left(2 s^{2} 2 p^{6} 3 s^{2}{ }^{1} \mathrm{~S}_{0}\right)$

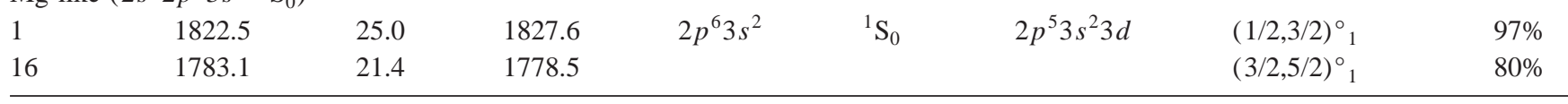

Al-like $\left(2 s^{2} 2 p^{6} 3 s^{2} 3 p^{2} \mathrm{P}_{1 / 2}^{\circ}\right)$

\begin{tabular}{lllllllll}
50 & 1803.9 & 6.8 & 1802.8 & $3 s^{2} 3 p$ & ${ }^{2} \mathrm{P}^{\circ}{ }_{1 / 2}$ & $2 p^{5} 3 s^{2} 3 p 3 d$ & $(1,3 / 2)_{3 / 2}$ & $90 \%$ \\
\hline \hline
\end{tabular}

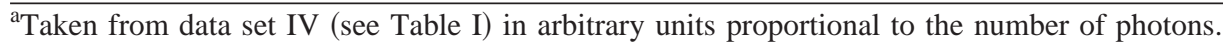

${ }^{\mathrm{b}}$ Average of data sets I-IV (see Table I). Estimated combined standard uncertainty is $3.5 \mathrm{eV}$.

${ }^{\mathrm{c}}$ Calculated with model (Sec. IV) for data set II. Intensities are in arbitrary units proportional to the number of photons/s.

${ }^{\mathrm{d}}$ Calculated with relativistic parametric potential method (HULLAC code).

${ }^{\mathrm{e}}$ Calculated with superposition-of-configurations method (COWAN code).

${ }^{\mathrm{f}}$ Identified first by Burkhalter [16] or Gordon [17].

trons in an open $2 p$ shell is relatively complicated and results in numerous closely spaced lines that we were not able to resolve experimentally. Because of the EBIT excitation mechanism, lines that have a strong electric dipole $(E 1)$ transition probability to the ground state dominate the spectrum. However, in the low-density environment (negligible collisional deexcitation) inside the EBIT E2 (electric quadrupole) and $M 1$ (magnetic dipole) transitions are also observable. The complexity of the spectra (for example, a spectrum of B-like $\mathrm{Kr}$ consists of more than one hundred $2 p^{6}-2 p^{5} 3 l$ lines that are calculated to be stronger than $1 / 200$ of the strongest line) demands additional tools for correct line identifications. We used two different theoretical calculations as an additional aid. The transition energies were calculated using the superposition-of-configurations method developed by R. D. Cowan [30]. For energy-level structure calculations we included all $n=2$ and $n=3$ configurations except the configurations with an open $1 s$ subshell, and $n=4$ configurations excluding all innershell excited configurations. The electrostatic and configuration interaction parameters were scaled to $90 \%$ of their $a b$ initio values whereas spin-orbit parameters were kept at $95 \%$ of their $a b$ initio values. It has been shown previously [30] that these estimates predict level energies closer to the experimental ones. Line intensities were calculated using a plasma model (see next section) that is based on a relativistic parametric potential calculation using the HULLAC code [31,32]. The qualitative behavior of the spectral lines under different experimental conditions was used to support the identifications.

\section{KR IONIZATION BALANCE}

\section{A. Basic Procedures}

The ionization fraction $f_{q}$ of the $\mathrm{Kr}$ ions with charge $q$ is given by 


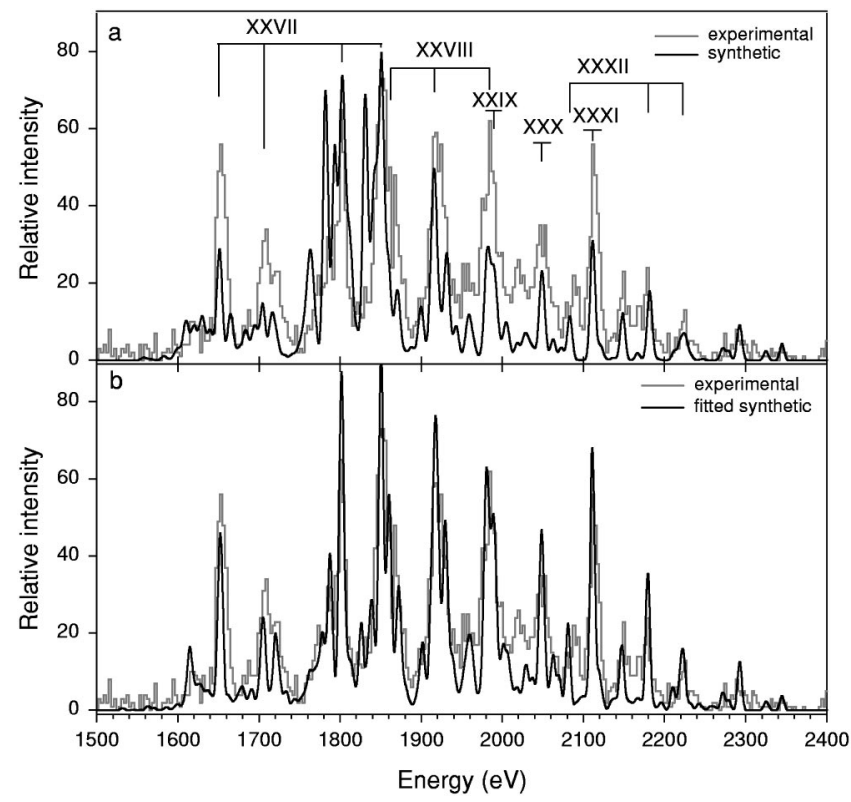

FIG. 1. Comparison of the experimental (set I, Table I) and fitted synthetic spectra of $\mathrm{Kr}$ calculated with the model described in Sec. IV. (a) equal charge state distribution. (b) nonequal (fitted) charge state distribution including charge-exchange processes. Roman numbers mark lines belonging to the corresponding $\mathrm{Kr}$ spectrum (e.g., XXVII marks Ne-like Kr etc.)

$$
\begin{aligned}
\frac{d f_{q}}{d t}= & n_{e}\left(I_{q-1} f_{q-1}-I_{q} f_{q}\right)+n_{e}\left[\left(R_{q+1}+D_{q+1}\right) f_{q+1}-\left(R_{q}\right.\right. \\
& \left.\left.+D_{q}\right) f_{q}\right]+n_{K r}\left(X_{q+1} f_{q+1}-X_{q} f_{q}\right)
\end{aligned}
$$

where $I_{q}, R_{q}, D_{q}$, and $X_{q}$ are the rates for electron-impact ionization, radiative recombination, dielectronic recombination, and charge-exchange recombination, respectively, for the charge state $q, n_{e}$ is the electron density and $n_{K r}$ is the density of neutral $\mathrm{Kr}$ atoms in the trap. The calculation of the various rates is discussed in more detail below. Equation 1 neglects charge exchange between ions and three-body recombination, the inverse of electron collisional ionization. The latter would scale as $n_{e}^{2}$ and, although insignificant for the EBIT beam, becomes important as the electron density increases.

The EBIT trap was emptied every $1.35 \mathrm{~s}$. As we will describe below, the relevant ionization and recombination rates are $\approx 100 \mathrm{~s}^{-1}$, allowing us to considerably simplify our model by assuming a steady-state ionization balance, i.e., putting the time derivatives in Eq. (1) equal to zero. Another important simplification comes from the observation that the ion-ion collision time in the trap, given by Ref. [33], is

$$
\tau_{i}=\frac{3}{4 \sqrt{\pi}} \frac{\sqrt{m_{i}}\left(k T_{i}\right)^{3 / 2}\left(4 \pi \epsilon_{0}\right)^{2}}{n_{i} q^{4} e^{4} \log \Lambda} \sim 10^{-3} \mathrm{~s}
$$

for an ion density $n_{i} \approx 6 \times 10^{14} \mathrm{~m}^{-3}$ (this is about $2 \%$ of the ion density required to fully compensate the space charge of the electron beam), where $m_{i}, T_{i}$, and $n_{i}$ are the ion mass, temperature, and density, $e$ is the elementary charge, and
$\Lambda=(4 \pi / 3) n_{e} \lambda_{D}^{3}$ with $\lambda_{D}=\sqrt{k T_{e} \epsilon_{0} / n_{e} q^{2} e^{2}}$ being the Debye length for electron temperature $T_{e}$ and density $n_{e}$. For typical conditions inside the EBIT, $\log \Lambda \approx 18$. This collision time is about an order of magnitude less than the ionization and recombination times, allowing us to put all ion temperatures at the same value. Note that when a light gas (e.g., oxygen) with significantly lower charge $q$ is introduced to provide evaporative cooling [22], this inequality is not met. The coolant gas may then have a different temperature than the trapped ions, as indeed it must have in order to cool the trap.

A relevant objection to the foregoing is that the Debye length is approximately an order of magnitude larger than the EBIT electron-beam radius, and the plasma parameter should more realistically be given by $\Lambda=(4 \pi / 3) n_{e} \lambda_{D} r_{b}^{2}$. The value of $\log \Lambda$ is then $\simeq 14$, and our conclusions still hold.

\section{B. Ionization, Recombination, and Loss Rates}

Cross sections for electron-impact ionization are taken from Ref. [34] for levels up to the $n=3$. The cross sections are essentially identical to those coming from the Lotz formula (see e.g., Ref. [35]). For the monoenergetic EBIT beam the rates are formed by simply multiplying the cross section by the electron velocity. Radiative recombination cross sections are taken in the Kramers' approximation (see e.g., Ref. [36]). We sum the radiative recombination cross section to each of the $n=2$ and $n=3$ configurations to arrive at the total cross section. Other workers [35] have used a formula due to [37] that sums the radiative recombination to $n=\infty$. This sum, however, includes only recombination to $s$ states.

In our model we neglect the dielectronic recombination because for the beam energies at which our spectra were taken (around $6 \mathrm{keV}$ and $12 \mathrm{keV}$ ), it is unlikely that any dielectronic resonances will exist in ions with ionization potentials in the range $1 \mathrm{keV}$ to $4 \mathrm{keV}$.

Charge-exchange recombination with neutral $\mathrm{Kr}$ atoms inside the trap are treated in a simple level crossing model. The potential of the ion-neutral system is written (in atomic units, where $\left.e=m_{e}=\hbar=1\right)$ as $V=-I_{q}+(q-1) / r=-I_{0}$, where $I_{q}$ and $I_{0}$ are the ionization potentials of the ion (after the charge-exchange process) and neutral (before charge exchange), respectively, and $r$ is the ion-neutral distance. Hence at a pseudocrossing $r_{c x}=(q-1) /\left(I_{q}-I_{0}\right)$, and the cross section is given by $\sigma_{c x}=\pi r_{c x}^{2}$, subject to the constraint that $r_{c x} \leqslant\left(2 q^{1 / 2}+1\right) / I_{0}$ (see Ref. [38] for details). During the EBIT runs described in this paper, it was not possible to get an accurate measurement of the neutral $\mathrm{Kr}$ density near the trapped ions (assuming that the charge exchange occurs between highly charged trapped ions and neighboring $\mathrm{Kr}$ neutrals) and so the above formulas were used to model the relative charge-exchange rates for the various $\mathrm{Kr}$ ions, with the absolute normalization left as a free parameter to vary when optimizing the model prediction to the observations.

The rates for ion reactions with electrons (impact ionization, radiative recombination) need to be modified to account for the time that the ions spend outside the electron beam in radial excursions. We neglect the azimuthal drift imposed on the ion orbits by the magnetic field. The potential in which the ions move is given by the solution to Laplace's equation 
in cylindrical symmetry, subject to the boundary condition that at a radius equal to the drift tube radius $\left(r_{d t}\right)$, the potential is $300 \mathrm{~V}$ (the voltage applied to form the electrostatic trap). Then the fraction of time that an ion spends inside the beam is given by

$$
f=\int_{0}^{r_{b}} \frac{d r}{v} /\left\{\int_{0}^{r_{b}} \frac{d r}{v}+\int_{r_{b}}^{r_{m}} \frac{d r}{v}\right\}
$$

where $r_{b}$ and $r_{m}$ are the electron-beam radius and maximum ion radius, and $v=v(r)$ is the ion velocity given by $m_{i} v^{2} / 2$ $=3 k T_{i} / 2-q e V$. Evaluating the integrals (using $r-r_{b} \ll r_{b}$ in the second) we arrive at the following expression for $f$ :

$$
f=\frac{\arcsin \sqrt{\eta}}{\arcsin \sqrt{\eta}+\sinh ^{-1}\left(\frac{r_{m} / r_{b}-1}{\sqrt{4+1 \eta}}\right)},
$$

where $\eta=(q e|Q / l|) / 6 \pi \epsilon_{0} k T_{i} \quad$ and $\quad r_{m}=r_{b} \exp \left[3 k T_{i} \pi \epsilon_{0} /\right.$ $\left.\times(q e|Q / l|)-\frac{1}{2}\right]$ with $Q / l$ being the charge per unit length of the electron beam. This fraction is implemented in our model neglecting the compensation of the trapped positive ions, consistent with an ion density of $6 \times 10^{14} \mathrm{~m}^{-3}$ assumed above.

The remaining process to be considered is that of ion escape from the trap. We follow the treatment of Ref. [35], assuming only radial escape to be important. They give an expression for the ion loss rate

$$
\frac{d N_{q}}{d t}=\nu_{\text {escape }} \Delta N_{q},
$$

where $N_{q}$ is the number of ions of charge $q$ and $\Delta N_{q}$ is the fraction of them with energy $E>q e V_{b}$, and $V_{b}$ is the trapping potential, i.e., the number of ions energetically allowed to escape from the trap. The escape rate $\nu_{\text {escape }}$ is given by

$$
\nu_{\text {escape }}=\frac{2}{r_{b}\left(r_{d t}-r_{b}\right)} \frac{k T_{i} \tau_{i} / m_{i}}{1+\left(\tau_{i} q e B / m_{i}\right)^{2}} \simeq 10^{3} \mathrm{~s}^{-1},
$$

which is derived from standard results for cross-field diffusion. For ions of charge $q$ from 26 to $32, \Delta N_{q} / N_{q}<10^{-2}$ giving a maximum loss rate of order $10 \mathrm{~s}^{-1}$. This is an order

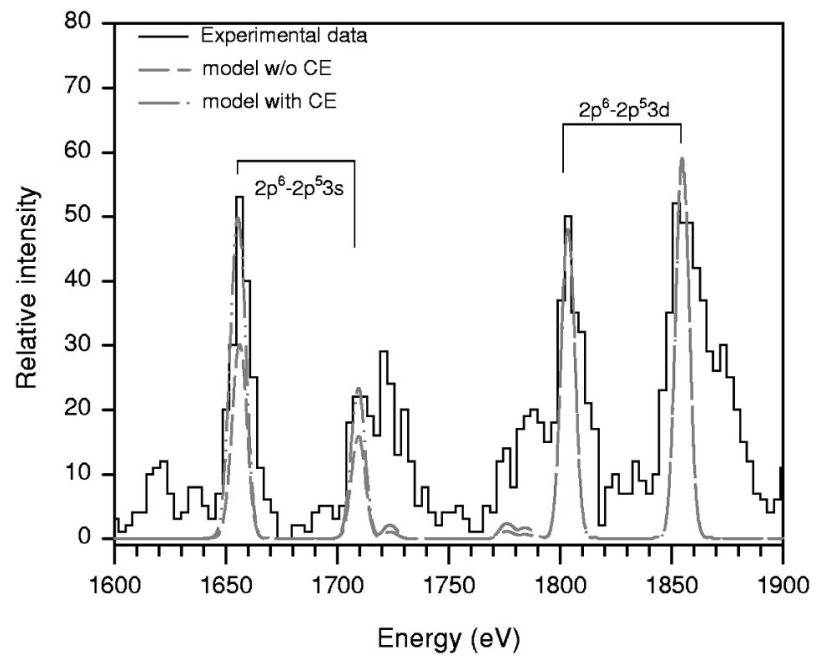

FIG. 2. Comparison of Ne-like $\mathrm{Kr} 2 p^{6}-2 p^{5} 3 s$ and $2 p^{6}-2 p^{5} 3 d$ lines (set IV, Table I). Charge exchange. Synthetic spectra are normalized using $2 p^{6}-2 p^{5} 3 d$ lines.

of magnitude lower than the collisional ionization and recombination rates and is neglected.

\section{Modeling and Fitting of Charge State Distributions}

A charge state distribution was fitted to the experimental data by the following procedure. Model spectra for each $\mathrm{Kr}$ ionization state (Al- to Be-like) were computed for the relevant EBIT beam parameters using the HULLAC suite of codes (Table III). These calculate the energy levels and radiative decay rates using a parametric potential, and the electron impact excitation cross sections in the distorted-wave approximation. The total synthetic spectrum is a sum of spectra of the ten (Al- to Be-like) different $\mathrm{Kr}$ ions where individual spectra are scaled with the relative charge state fraction. This total spectrum was fitted to the data using the charge state fractions as the fitting parameters on the assumption that electron-impact excitation was the sole excitation process, and a charge state distribution was obtained. Generally the agreement between experimental and fitted synthetic spectra was not satisfactory [Fig. 1(a)]. In particular, we noted a large discrepancy for the Ne-like and F-like

TABLE III. Configurations included for Kr ions in HULLAC calculations.

\begin{tabular}{ll}
\hline \hline Ion & \multicolumn{1}{c}{ Configurations } \\
\hline Li- & $2 s 2 p 3 s 3 p 3 d$ \\
Be- & $2 s^{2} 2 s 2 p 2 p^{2} 2 s 3 s 2 s 3 p 2 s 3 d$ \\
B- & $2 s^{2} 2 p 2 s 2 p^{2} 2 p^{3} 2 s^{2} 3 s 2 s^{2} 3 p 2 s^{2} 3 d 2 s 2 p 3 s 2 s 2 p 3 p 2 s 2 p 3 d$ \\
$\mathrm{C}-$ & $2 s^{2} 2 p^{2} 2 s 2 p^{3} 2 p^{4} 2 s^{2} 2 p 3 s 2 s^{2} 2 p 3 p 2 s^{2} 2 p 3 d$ \\
$\mathrm{~N}-$ & $2 s^{2} 2 p^{3} 2 s 2 p^{4} 2 p^{5} 2 s^{2} 2 p^{2} 3 s 2 s^{2} 2 p^{2} 3 p 2 s^{2} 2 p^{2} 3 d$ \\
$\mathrm{O}-$ & $2 s^{2} 2 p^{4} 2 s 2 p^{5} 2 p^{6} 2 s^{2} 2 p^{3} 3 s 2 s^{2} 2 p^{3} 3 p 2 s^{2} 2 p^{3} 3 d$ \\
$\mathrm{~F}-$ & $2 s^{2} 2 p^{5} 2 s 2 p^{6} 2 s^{2} 2 p^{4} 3 s 2 s^{2} 2 p^{4} 3 p 2 s^{2} 2 p^{4} 3 d 2 s 2 p^{5} 3 s 2 s 2 p^{5} 3 p$ \\
$\mathrm{Ne}-$ & $2 s^{2} 2 p^{6} 2 s^{2} 2 p^{5} 3 s 2 s^{2} 2 p^{5} 3 p 2 s^{2} 2 p^{5} 3 d 2 s 2 p^{6} 3 s 2 s 2 p^{6} 3 p 2 s 2 p^{6} 3 d$ \\
$\mathrm{Na}-$ & $2 s^{2} 2 p^{6} 3 s 2 s^{2} 2 p^{6} 3 p 2 s^{2} 2 p^{6} 3 d 2 s^{2} 2 p^{5} 3 s^{2} 2 s^{2} 2 p^{5} 3 s 3 p 2 s^{2} 2 p^{5} 3 s 3 d 2 s^{6} 3 s^{2} 2 s 2 p^{6} 3 s 3 p 2 s 2 p^{6} 3 s 3 d$ \\
$\mathrm{Mg}-$ & $2 s^{2} 2 p^{6} 3 s^{2} 2 s^{2} 2 p^{6} 3 s 3 p 2 s^{2} 2 p^{6} 3 s 3 d 2 s^{2} 2 p^{6} 3 p^{2} 2 s^{2} 2 p^{6} 3 p 3 d 2 s^{2} 2 p^{6} 3 d^{2} 2 s^{2} 2 p^{5} 3 s^{2} 3 p 2 s^{2} 2 p^{5} 3 s^{2} 3 d$ \\
& $2 s 2 p^{6} 3 s^{2} 3 p 2 s 2 p^{6} 3 s^{2} 3 d$ \\
\hline \hline
\end{tabular}


TABLE IV. $3 s / 3 d$ intensity ratios for some transitions in the Ne- and F-like Kr.

\begin{tabular}{lcccc}
\hline \hline Ion & Level $^{\mathrm{a}}$ & $\begin{array}{c}\text { Model } \\
\text { w/o CE }\end{array}$ & $\begin{array}{c}\text { Model } \\
\text { with CE }{ }^{\mathrm{b}}\end{array}$ & Exp. $^{\mathrm{c}}$ \\
\hline Ne-like & $3 s^{3} \mathrm{P}^{\circ}{ }{ }^{1}$ & 0.11 & 0.38 & 0.5 \\
& $3 s{ }^{1} \mathrm{P}^{\circ}$ & 0.44 & 0.60 & 0.9 \\
F-like & $3 s^{3} \mathrm{P}^{\circ}{ }_{1}$ & 0.24 & 0.34 & 0.4 \\
& $2 p^{4}\left({ }^{1} \mathrm{D}\right) 3 s(2,1 / 2)_{5 / 2}$ & 0.11 & 0.25 & 0.3 \\
& $2 p^{4}\left({ }^{3} \mathrm{P}\right) 3 s(2,1 / 2)_{5 / 2}$ & 0.12 & 0.43 & 0.4 \\
\hline \hline
\end{tabular}

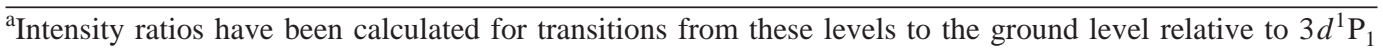
$\rightarrow$ ground level and $2 p^{4}\left({ }^{1} \mathrm{D}\right) 3 d(2,3 / 2)_{5 / 2} \rightarrow$ ground level transitions in $\mathrm{Ne}$ - and F-like $\mathrm{Kr}$, respectively.

${ }^{\mathrm{b}}$ Charge exchange (CE), see discussion in text.

${ }^{\mathrm{c}}$ Both experimental and theoretical data have been taken for $6 \mathrm{keV}$, low pressure (set IV, Table I) conditions.

Other spectra showed qualitatively similar behavior.

charge states, where substantially more intensity was observed in the $3 s \rightarrow 2 p$ lines relative to the $3 d \rightarrow 2 p$ lines of these ions (Fig. 2). The observation that these intensity ratios deviated more from the theory for the $6 \mathrm{keV} \mathrm{Kr}$ spectrum at high pressure than for the low pressure led us to suspect that charge exchange might play a role in exciting these lines.

The fitted ionization balance was then matched with a model with suitable choices for the normalization of the charge exchange recombination rates and the ion temperature in the trap. The emission spectrum from each ion was recalculated now including the effects of charge exchange, approximated by assuming that each level considered in the target ion was populated according to its statistical weight, followed by radiative cascading down. In this way the $3 \mathrm{~s}$ population in $\mathrm{Ne}$ - and F-like ions is boosted, since significant extra population cascades through these levels. In Fig. 2 the strongest $3 s$ and $3 d$ lines are shown together with the calculated spectra; the numerical values are presented in Table IV.

The spectra from each ion iterated in this way were refitted to the data treating the charge exchange rates and the temperature as additional fitting parameters. The final fitted charge state distribution is presented in Table $\mathrm{V}$.

\section{DISCUSSION}

From Figs. 1 and 2 it can be seen that our modeling approach gives a satisfactory account of the relative line intensities, at least for the strong well detected lines, over a wide range of charge states. We draw particular attention to the relative intensities in the Ne-like charge state $\mathrm{Kr}$ XXVII. From Fig. 2 it can be seen that irrespective of the inclusion of charge exchange in our model, the intensity ratio between the $2 p^{6}{ }^{1} S_{0}-2 p^{5} 3 d^{3} D_{1}$ and the $2 p^{6}{ }^{1} S_{0}-2 p^{5} 3 d^{1} P_{1}$ lines agrees with our calculations. This is quite different from the isoelectronic system Fe XVII, where discrepancies between theory and observations are well documented in the solar physics literature [39-44], and are becoming apparent in $\mathrm{x}$-ray observations of stellar coronae as well ( [45] and references therein). The ratios of $2 p^{6}-2 p^{5} 3 s$ lines to those from the $2 p^{6}-2 p^{5} 3 d$ configurations, which are also prob-

TABLE V. Fitted and modeled charge state distributions.

\begin{tabular}{|c|c|c|c|c|c|c|}
\hline \multirow[t]{2}{*}{ Ion } & \multicolumn{2}{|c|}{$12 \mathrm{keV}$} & \multicolumn{2}{|c|}{$6 \mathrm{keV}$ (low pressure) } & \multicolumn{2}{|c|}{$6 \mathrm{keV}$ (high pressure) } \\
\hline & $\mathrm{Fit}^{\mathrm{a}}$ & Model $^{\mathrm{b}}$ & Fit & Model & Fit & Model \\
\hline Li- & 0.010 & 0.021 & 0.024 & 0.011 & 0.003 & 0.0009 \\
\hline $\mathrm{Be}-$ & 0.097 & 0.070 & 0.10 & 0.051 & 0.060 & 0.008 \\
\hline B- & 0.17 & 0.14 & 0.16 & 0.13 & 0.091 & 0.032 \\
\hline $\mathrm{C}-$ & 0.19 & 0.20 & 0.17 & 0.21 & 0.096 & 0.085 \\
\hline $\mathrm{N}-$ & 0.16 & 0.20 & 0.13 & 0.23 & 0.14 & 0.15 \\
\hline $\mathrm{O}-$ & 0.14 & 0.16 & 0.14 & 0.18 & 0.11 & 0.20 \\
\hline F- & 0.087 & 0.010 & 0.10 & 0.11 & 0.17 & 0.21 \\
\hline $\mathrm{Ne}-$ & 0.066 & 0.060 & 0.085 & 0.055 & 0.17 & 0.17 \\
\hline $\mathrm{Na}-$ & 0.026 & 0.025 & 0.040 & 0.018 & 0.081 & 0.093 \\
\hline $\mathrm{Mg}-$ & 0.019 & 0.008 & 0.022 & 0.004 & 0.044 & 0.037 \\
\hline
\end{tabular}

${ }^{a}$ Fitted ionization balances are determined by least-squares fits to experimental data, using theoretical line intensities for each ion, iterated as described in the text to include the charge-exchange contribution.

${ }^{b}$ Model ionization balances are calculated by using the ionization and recombination rates described in the text, with the magnitude of the charge-exchange rates adjusted to give the best match to the "fitted" ionization balance. 
lematic in solar and astrophysical spectra, are harder for us to check experimentally because of the problems of charge exchange in our experiment enhancing the $2 p^{6}-2 p^{5} 3 s$ intensities.

These effects of charge exchange may however allow the measurement of state selective charge-exchange cross sections, relative to those for electron-impact excitation or ionization. As well as applications of neutral beam heating in tokamaks, we expect such data to be very useful in interpretation of cometary x-ray spectra [46-48]. Here, neutral material outgassing from the comet nucleus encounters highly charged ions in the solar wind. The ensuing charge-exchange collisions populate highly excited states of the solar wind ions, followed by $\mathrm{x}$-ray emission as these states radiatively decay. An astrophysically relevant experiment would require trapped ions of $\mathrm{C}, \mathrm{N}, \mathrm{O}$, or $\mathrm{Ne}$, and the admission to the EBIT chamber of the appropriate neutral gas, i.e., $\mathrm{H}_{2} \mathrm{O}, \mathrm{CO}_{2}$, etc.

Among our line identifications are several lines with potential for electron-density diagnostics. The density sensitivity arises because the relative populations among the different levels of the ground configurations varies with density. If this results in higher population accumulating in a level of higher angular momentum (i.e., the $2 s^{2} 2 p^{2} P_{3 / 2}$ in $\mathrm{Kr}$ XXXII, the $2 s^{2} 2 p^{2}{ }^{3} P_{2}$ in $\mathrm{Kr}$ XXXI, or the $2 s^{2} 2 p^{3}{ }^{2} D_{5 / 2}$ in $\mathrm{Kr}$ XXX) than the ground level, then excitations to levels among the $n=3$ configurations with higher angular momentum than are possible from the ground state alone, result. Hence new lines from these high angular momentum level states become visible at higher densities.

In Fig. 3(a) we show two line intensity ratios in Kr XXXII that vary as a result of $n=3, j=5 / 2$ levels becoming excited, calculated for an electron temperature of $2 \times 10^{7} \mathrm{~K}$. The electron densities over which this ratio varies is $10^{19}$ $-10^{21} \mathrm{~cm}^{-3}$, which is the relevant density range for high $\mathrm{Z}$ underdense plasma radiators. This should be very useful, since the density diagnostic available in the $\mathrm{Kr} K$-shell spectrum, the ratio of the $1 s^{2}{ }^{1} S_{0}-1 s 2 p^{3} P_{2}$ to either of the $1 s^{2}{ }^{1} S_{0}-1 s 2 p^{13} P_{1}$ lines only becomes sensitive at densities greater than $10^{21} \mathrm{~cm}^{-3}$, i.e., higher than is usually achieved in such experiments. One solution to this is to suspend pellets of material of slightly lower $Z$ in the Xe filled enclosure, so that the corresponding He-like transitions are sensitive in the right range. This would be most appropriate in the hottest regions of the $\mathrm{Xe}$ enclosure, since $\mathrm{Kr}$ and similar ions will be ionized beyond the B-like charge state, but elsewhere the B-like density diagnostic we discuss might be preferable. We note that previously discussed $L$ shell diagnostics in $\mathrm{Xe}[6,49]$ for the electron temperature are still dependent on the electron density, due to the density sensitivity of the dielectronic recombination process and the ionization balance itself in such experiments. The density diagnostic we identify is much less dependent on the electron temperature, and would go some way towards resolving such ambiguities.

Further density sensitive line ratios at the same electron temperature in $\mathrm{Kr}$ XXXI and $\mathrm{Kr}$ XXX are plotted in Fig. 3(b), which may have applications to tokamak plasmas with density in the range $10^{12}-10^{14} \mathrm{~cm}^{-3}$.
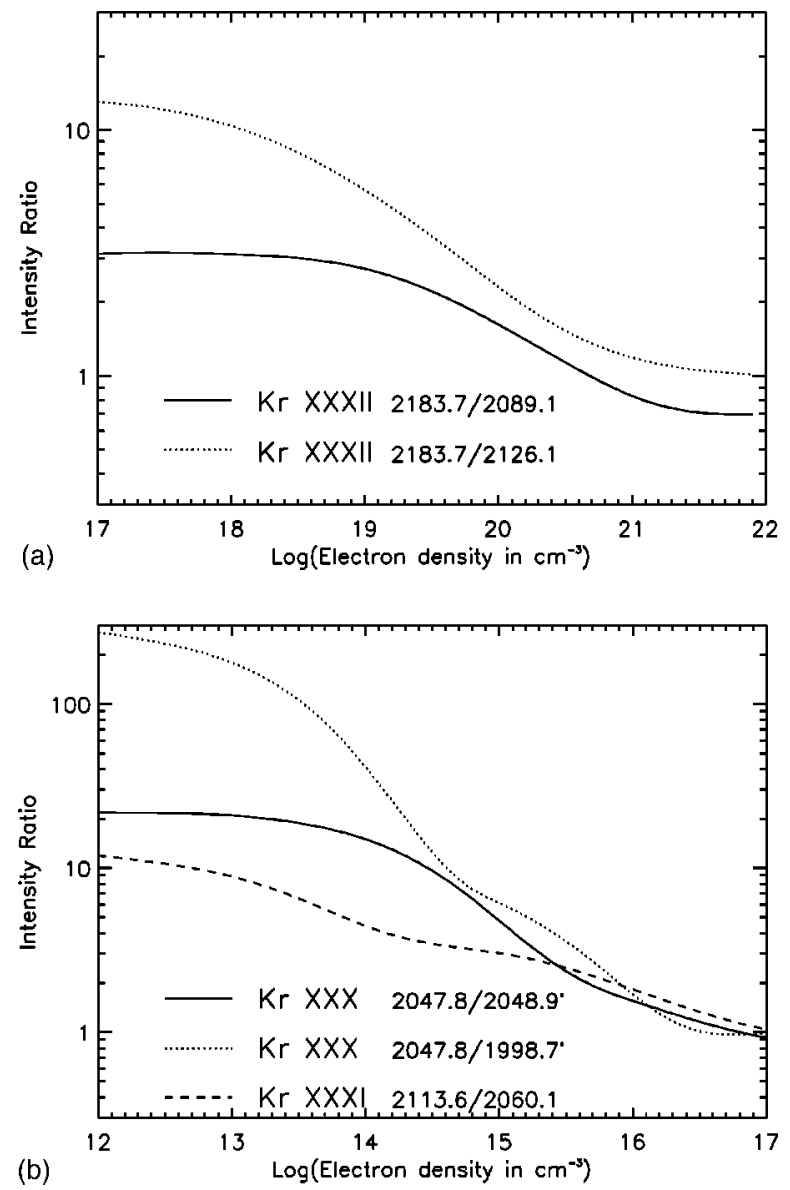

FIG. 3. Electron-density $\left(\log _{10} n_{e}\right)$ dependence of line intensity ratios at electron temperature $T_{e}=2 \times 10^{7} \mathrm{~K}$ calculated with a model described in Sec. IV for selected transitions in Kr XXXXXXII (identified in Table II).

\section{CONCLUSION}

We have demonstrated the value of using an EBIT plasma model for the analysis of complex and only partially resolved $\mathrm{x}$-ray spectra obtained with a broadband high-resolution $\mathrm{x}$-ray detector. This approach can be applied in the determination of numerous physical characteristics of the EBIT plasma, such as measurements of important reaction rates and the validation of diagnostic line ratios. In this paper we have identified many new lines in the spectra of $L$-shell $\mathrm{Kr}$ ions, some with diagnostic potential applicable in other fields of plasma physics, and have made progress towards understanding conditions in the EBIT trap itself.

\section{ACKNOWLEDGMENTS}

The authors thank D. J. Alderson for his technical support. I.K. acknowledges support from the Swedish Foundation for Cooperation in Research and Higher Education (STINT). J.M.L. was supported by NASA Contract W 19353 (Applied Information Systems Research Program) and ONR/NRL 6.1 Research Option "Solar Magnetism and the Earth's Environment." 
[1] J. M. Laming, C. A. Back, C. D. Decker, J. Grun, U. Feldman, J. F. Seely, and J. F. Davis, Phys. Rev. E (to be published).

[2] C. A. Back, J. Grun, C. Decker, L. J. Suter, J. Davis, O. L. Landen, R. Wallace, W. W. Hsing, J. M. Laming, U. Feldman, M. Miller, and C. Wuest, Phys. Rev. Lett. (to be published).

[3] H. Fiedorowicz, A. Bartnik, P. Parys, and Z. Patron, Inst. Phys. Conf. Ser. 130, 515 (1992).

[4] M. J. May, M. Finkenthal, V. Soukhanovskii, D. Stutman, H. W. Moos, D. Pacella, G. Mazzitelli, K. Fournier, W. H. Goldstein, and B. Gregory, Rev. Sci. Instrum. 70, 375 (1999).

[5] M. Bitter, H. Hsuan, K. W. Hill, and M. Zarnstorff, Phys. Scr. T47, 87 (1993).

[6] C. J. Keane, B. A. Hammel, A. L. Osterheld, and D. R. Kania, Phys. Rev. Lett. 72, 3029 (1994).

[7] J. D. Gillaspy, Phys. Scr. T65, 168 (1996).

[8] B. Edlén, in Handbuch der Physik, edited by S. Flügge (Springer-Verlag, Berlin, 1964), p. 148.

[9] S. Bandler, E. Silver, H. Schnopper, S. Murray, M. Barbera, N. Madden, D. Landis, J. Beeman, E. Haller, and G. Tucker, Nucl. Instrum. Methods Phys. Res. A 444, 273 (2000).

[10] E. Silver, H. Schnopper, S. Bandler, S. Murray, N. Madden, D. Landis, J. Beeman, E. Haller, M. Barbera, G. Tucker, J. Gillaspy, E. Takacs, and J. Porto, Nucl. Instrum. Methods Phys. Res. (to be published).

[11] M. J. May, M. Finkenthal, V. Soukhanovskii, D. Stutman, H. W. Moos, D. Pacella, G. Mazzitelli, K. Fournier, W. Goldstein, and B. Gregory, Rev. Sci. Instrum. 70, 375 (1999).

[12] M. J. May, K. B. Fournier, D. Pacella, H. Kroegler, J. E. Rice, B. Gregory, M. Finkenthal, H. W. Moos, G. Mazzitelli, and W. H. Goldstein, Phys. Rev. E 61, 3042 (2000).

[13] J. P. Buchet, M. C. Buchet-Poulizac, A. Denis, J. Desesquelles, M. Druetta, S. Martin, D. Lecer, E. Luc-Koenig, and J. F. Wyart, Nucl. Instrum. Methods Phys. Res. B 31, 177 (1988).

[14] E. Hinnov, Phys. Rev. A 14, 1533 (1976).

[15] J. F. Wyart and TFR Group, Phys. Scr. 31, 539 (1985).

[16] P. G. Burkhalter, J. Shiloh, A. Fisher, and R. D. Cowan, J. Appl. Phys. 50, 4532 (1979).

[17] H. Gordon, M. G. Hobby, N. J. Peacok, and R. D. Cowan, J. Phys. B 12, 881 (1979).

[18] J. D. Gillaspy, J. R. Roberts, C. M. Brown, and U. Feldman, in Proceedings of the VIth International Conference on the Physics of Highly Charged Ions, edited by P. Richard, M. Stockli, C. L. Cocke, and C. D. Lin (AIP press, New York, 1993), Vol. 274, p. 682.

[19] J. D. Gillaspy, Phys. Scr. T71, 99 (1997).

[20] E. Silver, H. Schnopper, S. Bandler, N. Brickhouse, S. Murray, M. Barbera, E. Takacs, J. Gillaspy, J. Porto, I. Kink, N. Madden, D. Landis, J. Beeman, and E. Haller, Astrophys. J. (to be published).

[21] F. G. Serpa, E. W. Bell, E. S. Meyer, J. D. Gillaspy, and J. R. Roberts, Phys. Rev. A 55, 1832 (1997).

[22] B. M. Penetrante, J. N. Bardsley, M. A. Levine, D. A. Knapp, and R. E. Marrs, Phys. Rev. A 43, 4873 (1991).

[23] E. Silver, H. Schnopper, and R. Ingram, US Patent Application No. 09064476, 1998.

[24] H. Schnopper, E. Silver, R. Ingram, F. Christensen, A. Hussain, M. Barbera, S. Romaine, A. Collura, A. Kenter, S. Bandler, and S. Murray, Proc. SPIE 3766, 350 (1999).

[25] B. Edlén, Rep. Prog. Phys. 26, 181 (1963).

[26] F. Tyrén, Nova Acta Regiae Soc. Sci. Ups. 12, 7 (1940).

[27] N. J. Peacock, R. J. Speer, and M. G. Hobby, J. Phys. B 2, 798 (1969).

[28] J. D. Garcia and J. E. Mack, J. Opt. Soc. Am. 55, 654 (1965).

[29] L. Engström, Lund Reports on Atomic Physics, LRAP-232, University of Lund, 1998.

[30] R. D Cowan, The Theory of Atomic Structure and Spectra (University of California, Berkeley, 1981).

[31] A. Bar-Shalom and M. Klapisch, Comput. Phys. Commun. 50, 375 (1988).

[32] A. Bar-Shalom, M. Klapisch, and J. Oreg, Phys. Rev. A 38, 1773 (1988).

[33] D. B. Melrose, Instabilities in Space and Laboratory Plasmas (Cambridge University, Cambridge, 1986).

[34] D. L. Moores, L. B. Golden, and D. H. Sampson, J. Phys. B 13, 385 (1980).

[35] I. V. Kalagin, D. Küchler, V. P. Ovsyannikov, and G. Zschornack, Plasma Sources Sci. Technol. 7, 441 (1998).

[36] H. A. Bethe and E. E. Salpeter, Quantum Mechanics of Oneand Two-Electron Atoms (Springer-Verlag, Berlin, 1957).

[37] Y. S. Kim and R. H. Pratt, Phys. Rev. A 27, 2913 (1983).

[38] R. Mann, F. Folkmann, and H. F. Beyer, J. Phys. B 14, 1161 (1981).

[39] A. K. Bhatia and G. A. Doschek, At. Data Nucl. Data Tables 52, 1 (1992).

[40] K. J. H. Phillips, C. J. Greer, A. K. Bhatia, and F. P. Keenan, Astrophys. J. 469, L57 (1996).

[41] J. L. R. Saba, J. T. Schmelz, A. K. Bhatia, and K. T. Strong, Astrophys. J. 510, 1064 (1999).

[42] A. K. Bhatia and S. O. Kastner, Astrophys. J. 516, 482 (1999).

[43] G. V. Brown, P. Beiersdorfer, D. A. Liedahl, K. Widman, and S. M. Kahn, Astrophys. J. 502, 1015 (1998).

[44] L. W. Acton, M. E. Bruner, W. A. Brown, B. C. Fawcett, W. Schweizer, and R. J. Speer, Astrophys. J. 291, 865 (1985).

[45] J. M. Laming, I. Kink, E. Takacs, J. V. Porto, J. D. Gillaspy, E. H. Silver, H. W. Schnopper, S. R. Bandler, N. S. Brickhouse, S. S. Murray, M. Barbera, A. K. Bhatia, G. A. Doschek, N. Madden, D. Landis, J. Beeman, and E. E. Haller, Astrophys. J. Lett. (to be published).

[46] V. Kharchenko and A. Dalgarno, J. Geophys. Res. 105, 18351 (2000).

[47] J. B. Greenwood, I. D. Williams, S. J. Smith, and A. Chutjian, Astrophys. J. Lett. 533, L175 (2000).

[48] T. E. Cravens, Geophys. Res. Lett. 24, 105 (1997).

[49] B. H. Failor, J. C. Fernandez, B. H. Wilde, A. L. Osterheld, J. A. Cobble, and P. L. Gobby, Phys. Rev. E 59, 6053 (1999). 